\title{
Quality Improvement for Portal Vein Embolization
}

\author{
Alban Denys • Pierre Bize • Nicolas Demartines • \\ Frederic Deschamps · Thierry De Baere
}

Received: 5 October 2009/Accepted: 5 October 2009/Published online: 4 February 2010

(C) Springer Science+Business Media, LLC and the Cardiovascular and Interventional Radiological Society of Europe (CIRSE) 2010

\section{Introduction}

Major hepatectomy carries a significant risk of mortality. In patients with normal liver bearing metastases, mortality after major hepatectomy ranges from $0.5 \%$ to $4 \%$, but in patients [1] with chronic liver disease, such as cholestatic or cirrhotic liver, mortality increases to $4 \%$ to $12 \%[2,3]$. The main cause of mortality as well as postoperative morbidity after major hepatic resection is liver insufficiency, often due to an insufficient liver remnant volume $[4,5]$. It has been demonstrated from animal experiments and clinical data that redirection of the portal flow toward a part of the liver will induce hypertrophy of this part. This redirection of portal flow can be obtained by surgical ligation or by percutaneous embolization (PVE). Today, PVE is preferred to surgical ligation to avoid additional surgery. However, when surgery is performed, usually for resection of the primary tumor, and portal vein flow redistribution is required, no clear recommendations can be given regarding whether it is preferable to carry out percutaneous PVE in a second step or ligation at the time of surgery. Some studies have reported greater hypertrophy

\footnotetext{
A. Denys $(\bowtie) \cdot$ P. Bize

Department of Radiology and Interventional Radiology, Centre Hospitalier Universitaire Vaudois, University of Lausanne, 1011 Lausanne, Switzerland

e-mail: albandenys@orange.fr; Alban.Denys@chuv.ch

N. Demartines

Department of Visceral Surgery, Centre Hospitalier

Universitaire Vaudois, University of Lausanne,

1011 Lausanne, Switzerland

F. Deschamps · T. De Baere

Department of Interventional Radiology,

Institut Gustave Roussy, Villejuif, France
}

after PVE [6], whereas others have reported no differences in hypertrophy [7]. The aim of PVE is to (1) preoperatively increase the volume of the future liver remnant to enable surgery and (2) to decrease postoperative morbidity when the only contraindication to surgery is an initially insufficient remnant liver.

\section{Definitions}

Future liver remnant (FLR) is the liver that will be left in place after surgery and that was not targeted by embolization. The FLR must hypertrophy after portal vein embolization (PVE). Most teams wait 4 weeks before surgery. FLR hypertrophy must be measured by way of computed axial tomography (CAT) examination after injection of iodine with volumetric measurements of the FLR segments, with the results compared with the measurements performed before PVE using the same technique.

Hypertrophy can be quantified as FLR hypertrophy, which is defined as the difference between FLR after a waiting period from 3 to 6 weeks after PVE minus FLR before PVE divided by FLR before PVE. The waiting period must be long enough to allow hypertrophy and as short as possible to avoid tumor growth, which precludes surgery. Hypertrophy can also be quantified by increased FLR ratio. The FLR ratio is defined as (FLR volume-tumor in the FLR)/(total liver volume-total tumor volume) [8].

Technical success of PVE is defined by a complete occlusion of portal branches feeding the future resected liver segments. Branches of the FLR must be patent with hepatopetal flow. In the late phase of control portography, parenchymography must be visible only in the FLR.

Clinical success is considered to occur when the patient reaches the volumetric criteria for liver resection. 
Major hepatic resection (or major hepatectomy) is defined as a resection of at least four of eight segments of the liver. Right hepatectomy is defined as a resection of segments V to VIII. Extended right hepatectomy additionally includes segment IV.

Resection rate is defined as the number of embolized patients that will ultimately be resected.

\section{Materials and Methods}

\section{Patient Selection}

Patients must be candidates for major hepatectomy or major hepatectomy associated with radiofrequency ablation on the FLR. This decision should be made in the context of a tumor board meeting that includes surgeons, hepatologists, oncologists, and interventional radiologists. The only contraindication to liver resection must be the initially insufficient volume of the FLR. PVE indication is determined by the liver volumetry obtained from liver CAT [9].

Liver volumetry is calculated manually from CAT slices after injection of contrast media, preferably in the portal or equilibrium phase to opacify all of the portal and hepatic veins. Slice thickness $\leq 5 \mathrm{~mm}$ is recommended. The FLR is determined according to the future resection plane in collaboration with the surgical team. If radiofrequency ablation (RFA) is planned on a tumor in the FLR, the expected volume of RFA must be taken into consideration and subtracted from the FLR volume. Tumor tissue must not be taken into consideration for volumetric evaluation because volumetry must evaluate liver tissue only.

Blood tests-including liver enzyme, bilirubin level, partial thromboplastin time, prothrombin time (PT), and platelet count must be obtained before PVE. Criteria to propose PVE are different according to the liver status and to the tumor involvement as follows:

\section{Patients with Tumors that Developed in Normal Underlying Liver Parenchyma}

PVE is recommended when the FRL-to-total liver ratio is $<25$ to 30 [7, 10, 11]. The indication of PVE can be extended to a $40 \%$ FLR ratio in patients having received chemotherapy or showing abnormal indocyanine green test results (or other abnormal liver function tests) [10, 12, 13].

\section{Patients with Tumors that Develop in Chronic Liver Disease and Cirrhosis States}

In such cases, the decision is based either on liver volume or on liver volume plus estimation of overall liver function by indocyanin green retention rate at $15 \mathrm{~min}$ (ICCG 15).
An FRLR of $40 \%$ is recommended when the ICCG 15 is between $10 \%$ and $20 \%$. When the ICCG 15 is $>20 \%$, an FRLR of $50 \%$ is recommended [12-14].

Patients with Tumors Invading the Biliary Tree Associated with Cholestasis

Because biliary obstruction has impaired liver regeneration and hypertrophy, the biliary tree of the FRL must be drained first, and PVE can be performed secondarily. The indication is an FRLR $<40 \%$ [15].

Contraindication for PVE [11]

PVE is contraindicated in the following types of patients:

1. Tumors invading the portal vein

(a) Portal hypertension (blocked to free hepatic vein pressure gradient $>12 \mathrm{mmHg}$ )

2. Coagulation disorders (PT $<60 \%$, platelet count $<50$ $\mathrm{G} / 1)$

(a) Even if previous transarterial chemoembolization (TACE) may improve PVE results [16], a minimum of 3-week delay between TACE and PVE is recommended.

\section{Patient Information to be Given Before Treatment}

Patients should be informed that this procedure is not an antitumoral treatment but a treatment made to increase safety or to enable a surgical procedure. Minor complications are encountered in $20 \%$ to $25 \%$ of cases and are mainly associated with slight fever and abdominal discomfort and pain. Major complications are infrequent and mainly include infection and subcapsular hematoma, hemobilia, and portal vein thrombosis ( $<2 \%$ of cases). Mortality due to PVE has not been reported.

When tumors (usually small nodules) are present in the nonembolized lobe, it must be explained to the patient that those lesions might increase in size more quickly due to PVE [17]. Patients must also be told that the efficacy of the procedure can be estimated approximately 4 weeks after PVE by way of CAT with injection of contrast media and liver volumetry.

\section{Embolization Method}

Access to the portal system should be done under ultrasound guidance to puncture a peripheral branch [8]. Access can be obtained by way of controlateral approach (i.e., puncture of the left portal branch and embolization of the 
right portal branches) or ispsilateral approach (puncture of the right portal branch to embolize right portal branches. The advantage of the controlateral approach is easier catheterization, but there is a risk of damage to the FLR.

Five-French materials (catheter or introductory sheath) are usually recommended. The catheter should be placed at the splenomesenteric confluence to perform a portography to visualize portal anatomy, including its variations, and to localize segment IV branches. Measurement of portal pressure is not routinely performed in patients with normal liver. In cirrhotic patients, measuring the portal and central venous pressures is useful to determine whether the patient has a portostemic gradient $>12 \mathrm{mmHg}$ in, which case the patient is at major risk of perisurgical complications [18, 19]. These patients are not eligible for PVE.

The aim of embolization is complete obstruction of the targeted branches and redistribution of flow to the FLR branches only. Final portography is mandatory to verify this objective. A final pressure measurement should be obtained at the end of the procedure in patients with chronic liver disease to document portal pressure increase, which is usually approximately $3 \mathrm{mmHg}$. Embolization of segment IV branches is recommended in patients with tumors who are undergoing extended right hepatectomy. However, if embolization of that segment causes risk of reflux into the portal branch of the FRL, such embolization must not be performed because any major reflux into FRL portal branches might preclude surgery.

Various embolic materials have been used. Some products are not recommended due to reported recanalization or lower induced hypertrophy as follows:

\section{Gelfoam}

Gelfoam is associated with a high rate of portal vein recanalization and seems less efficient than other products [8].

\section{Nonspherical Polyvinyl Alcohol Particles}

Polyvinyl alcohol particles (PVA) have been used but are less efficient than spherical particles [20].

\section{Alcohol}

Direct intraportal injection has been described. Although efficient, it is hard to control and has been associated with significant morbidity (liver necrosis, portal vein thrombosis) [21].

\section{Drug-Loadable Embols}

These have not been reported in PVE and cannot be recommended.
Recommended products $[14,20,22]$ include the following:

\section{Mixture of n-Butyl-cyanoacrylate and Iodized Oil}

This has been described extensively as showing good results and low morbidity. Usually a mixture of one part $n$ butyl-cyanoacrylate (NBCA) to one or to parts lipiodol is used. Injections of small aliquots in between abundant flushing with nonionic liquid, such as dextran or glucose $5 \%$, is the most commonly reported technique.

\section{Spherical Microparticles}

These are associated with coil embolization, which is mostly described in North American reports, and have been reported to be superior to nonspherical PVA. It seems as efficient as NBCA, although it has never been compared in randomized trials. Most teams start with 300- to 500- $\mu \mathrm{m}$ particles and finish with 700- to 900- $\mu \mathrm{m}$ particles. Coils are used at the end of the procedure to allow for complete occlusion of the proximal trunk. It is advisable to avoid all too proximal occlusions and rather leave $1 \mathrm{~cm}$ unembolized segment of the right portal branch to facilitate surgical ligation at the time of liver resection.

\section{Association of Fibrin Glue with Iodized Oil}

This has mostly been described in Japan and has the drawback of requiring special catheters that are only available in Asia.

\section{Medication and Periprocedural Cure}

PVE can be carried out as an outpatient procedure when conscious sedation is used. A 1- or 2-day hospital stay is recommended, as reported by most teams using either conscious sedation associated with local anesthesia or general anesthesia.

There is no consensus on the use of antibiotics, and the type of antibiotics and the length of treatment vary from one report to another [11], except in patients with an associated biliary procedure. Because little to no liver tissue necrosis is found after PVE if an antibiotic is given, it must be administered for a very short period of time, from a single dose to $48 \mathrm{~h}$. Mild to moderate abdominal pain after PVE has been reported in $20 \%$ to $30 \%$ of patients and is usually easily controlled by oral analgesia. Use of pure alcohol as embolic material is associated with more severe abdominal pain.

Biologic tests are not mandatory after the procedure. Slightly increased aspartate aminotransferase and alanine aminotransferase levels with a peak at day 3 after the 
procedure have been reported, but these are without clinical significance.

\section{Results [14]}

The technical success rate should be close to $100 \%$. Few cases of failures or repeated procedures have been reported in the literature. The resection rate should be approximately $85 \%$. This rate may decrease to $70 \%$ in the case of cirrhotic patients. Reasons for nonresection are tumor progression, peritoneal metastases, or unsuspected metastases discovered at laparotomy. Absence of hypertrophy is rare, $<10 \%$ in metastatic liver, but it can reach $20 \%$ in cirrhotic patients.

In patients with normal liver and liver metastases, the increase of the FLR ratio is between $8 \%$ and $25 \%$, and regeneration is always observed after PVE. In cirrhotic patients, PVE fails to induce left-lobe hypertrophy in $20 \%$ of cases. Increased rate of FLR ratio in this population is slightly lower, between $6 \%$ and $20 \%$.

Recent studies have demonstrated that hypertrophy is inversely proportional to the FRL ratio before PVE, meaning that the smaller FRL before PVE will have the larger hypertrophy. Consequently there is no lower limit for the FRL ratio to perform PVE.

\section{Complications [14, 23]}

In terms of complications (Table 1), a rate of minor complications $(20 \%$ to $25 \%)$ is considered acceptable. With major complications, this rate should be $<5 \%$ and should not preclude further liver resection. Except for inadvertent embolization, most complications will occur in the punctured lobe, which is an argument in favour of a homolateral

Table 1 PVE procedure

\begin{tabular}{lll}
\hline & $\begin{array}{l}\text { Mean } \\
\text { reported } \\
\text { rate (\%) }\end{array}$ & $\begin{array}{l}\text { Suggested } \\
\text { procedure } \\
\text { threshold (\%) }\end{array}$ \\
\hline Minor complications & & \\
$\quad$ Abdominal pain & 20 & 40 \\
Fever & 25 & 50 \\
Nausea & 2.5 & 5 \\
Embolic material displaced & 0.2 & 0.4 \\
$\quad$ in FRL without portal thrombosis & & \\
Major complications & & \\
$\quad$ Liver abscess & 0.3 & 0.6 \\
Cholangitis & 0.2 & 0.4 \\
Main or left portal vein thrombosis & 0.2 & 0.4 \\
Subcapsular hematoma & 0.2 & 0.4 \\
\hline
\end{tabular}

approach. However, complication rates for homolateral and controlateral approaches are the same. Reported in the literature as the only factor increasing complications is puncture of the posterior segment versus puncture of the anterior segment [24], thus advocating puncture of the anterior segment when compatible with the location of the PVE to be performed.

\section{References}

1. Nordlinger B, Peschaud F, Malafosse R (2003) Resection of liver metastases from colorectal cancer-how can we improve results? Colorectal Dis 5:515-517

2. Farges O, Belghiti J, Kianmanesh R et al (2003) Portal vein embolization before right hepatectomy: prospective clinical trial. Ann Surg 237:208-217

3. Fong Y, Fortner J, Sun RL, Brennan MF, Blumgart LH (1999) Clinical score for predicting recurrence after hepatic resection for metastatic colorectal cancer: analysis of 1001 consecutive cases. Ann Surg 230:309-318

4. Abdalla EK, Barnett CC, Doherty D, Curley SA, Vauthey JN (2002) Extended hepatectomy in patients with hepatobiliary malignancies with and without preoperative portal vein embolization. Arch Surg 137:675-680

5. Yigitler C, Farges O, Kianmanesh R, Regimbeau JM, Abdalla EK, Belghiti J (2003) The small remnant liver after major liver resection: how common and how relevant? Liver Transpl 9(9):S18-25

6. Broering DC, Hillert C, Krupski G et al (2002) Portal vein embolization vs. portal vein ligation for induction of hypertrophy of the future liver remnant. J Gastrointest Surg 6:905-913

7. Capussotti L, Muratore A, Baracchi F et al (2008) Portal vein ligation as an efficient method of increasing the future liver remnant volume in the surgical treatment of colorectal metastases. Arch Surg 143:978-982

8. de Baere T, Roche A, Elias D, Lasser P, Lagrange C, Bousson V (1996) Preoperative portal vein embolization for extension of hepatectomy indications. Hepatology 24:1386-1391

9. Vauthey JN, Chaoui A, Do KA et al (2000) Standardized measurement of the future liver remnant prior to extended liver resection: methodology and clinical associations. Surgery 127:512-519

10. Ferrero A, Vigano L, Polastri R et al (2007) Postoperative liver dysfunction and future liver remnant: where is the limit? Results of a prospective study. World J Surg 31:1643-1651

11. Madoff DC, Abdalla EK, Vauthey JN (2005) Portal vein embolization in preparation for major hepatic resection: evolution of a new standard of care. J Vasc Interv Radiol 16:779-790

12. Azoulay D, Castaing D, Krissat J et al (2000) Percutaneous portal vein embolization increases the feasibility and safety of major liver resection for hepatocellular carcinoma in injured liver. Ann Surg 232:665-672

13. Kubota K, Makuuchi M, Kusaka K et al (1997) Measurement of liver volume and hepatic functional reserve as a guide to decision-making in resectional surgery for hepatic tumors. Hepatology $26: 1176-1181$

14. Abulkhir A, Limongelli P, Healey AJ et al (2008) Preoperative portal vein embolization for major liver resection: a meta-analysis. Ann Surg 247:49-57

15. Nimura Y, Kamiya J, Kondo S et al (2000) Aggressive preoperative management and extended surgery for hilar cholangiocarcinoma: Nagoya experience. J Hepatobiliary Pancreat Surg 7:155-162 
16. Ogata S, Belghiti J, Farges O, Varma D, Sibert A, Vilgrain V (2006) Sequential arterial and portal vein embolizations before right hepatectomy in patients with cirrhosis and hepatocellular carcinoma. Br J Surg 93:1091-1098

17. Elias D, De Baere T, Roche A, Ducreux M, Leclere J, Lasser P (1999) During liver regeneration following right portal embolization the growth rate of liver metastases is more rapid than that of the liver parenchyma. Br J Surg 86:784-788

18. Bruix J, Castells A, Bosch J et al (1996) Surgical resection of hepatocellular carcinoma in cirrhotic patients: prognostic value of preoperative portal pressure. Gastroenterology 111:1018-1022

19. Farges O, Malassagne B, Flejou JF, Balzan S, Sauvanet A, Belghiti J (1999) Risk of major liver resection in patients with underlying chronic liver disease: a reappraisal. Ann Surg 229:210-215

20. Madoff DC, Abdalla EK, Gupta S et al (2005) Transhepatic ipsilateral right portal vein embolization extended to segment IV: improving hypertrophy and resection outcomes with spherical particles and coils. J Vasc Interv Radiol 16(2 Pt 1):215-225

21. Shimamura T, Nakajima Y, Une Y et al (1997) Efficacy and safety of preoperative percutaneous transhepatic portal embolization with absolute ethanol: a clinical study. Surgery 121:135141

22. de Baere T, Denys A, Paradis V (2009) Comparison of four embolic materials for portal vein embolization: Experimental study in pigs. Eur Radiol [Epub ahead of print]

23. Di Stefano DR, de Baere T, Denys A et al (2005) Preoperative percutaneous portal vein embolization: evaluation of adverse events in 188 patients. Radiology 234:625-630

24. Kodama Y, Shimizu T, Endo H, Miyamoto N, Miyasaka K (2002) Complications of percutaneous transhepatic portal vein embolization. J Vasc Interv Radiol 13:1233-1237 